\title{
La gestion intégrée des matières résiduelles : revue des outils favorisant l'écologie industrielle
}

\author{
Gabriel Rojo, \\ ingénieur junior, doctorant à la Station expérimentale de procédés pilotes en environnement (STEPPE), \\ École de technologie supérieure de Montréal \\ Pour tout contact : gabriel.rojo. I@ens.etsmtl.ca
}

\section{Résumé}

Devant la nécessité d'instaurer les principes du développement durable dans nos sociétés industrialisées, et face aux volumes grandissants de résidus industriels générés, il s'avère essentiel de mettre en place des mécanismes pour soutenir la gestion de ces matières résiduelles (Thomas et al., 2005). Au cours des trois dernières décennies, plusieurs outils ont été développés pour appuyer la prise de décisions lors de cette gestion. Toutefois, des lacunes ont été relevées suite à l'application de ces outils, en raison de l'impossibilité d'effectuer, de façon simple et efficace, des comparaisons ou des simulations de scénarios dans le but d'analyser le comportement des systèmes de gestion (Harrison et al., 200I). L'analyse des impacts environnementaux et celle des coûts sont les principales fonctions des outils actuellement disponibles, et le comportement systémique découlant des décisions prises est ignoré (Wang et al., 1996). En ce sens, de nouveaux outils devront être élaborés afin de proposer des solutions plus durables pour la gestion des matières résiduelles.

\section{Mots clés :}

gestion intégrée, matières résiduelles, résidus industriels, écologie industrielle, aide à la prise de décisions, développement durable, analyse systémique, outil prévisionnel

Considering the necessity to establish principles of sustainable development within our industrialized society, and faced with growing volumes of industrial waste, it became essential to set up mechanisms to support the management of these residues (Thomas et al., 2005). Throughout the last three decades, many tools have been elaborated to assist decision making. However, some deficiencies were exposed after employing these tools because of the impossibility to execute, in a simple and effective way, comparisons and simulations of scenarios in order to analyse the behaviour of management systems (Harrison et al., 200 I). Generally, the examination of environmental impacts and costs are the main functions imputed to current tools. Furthermore, the systemic behaviour stem from the decisions taken is totally ignored (Wang et al., 1996). Consequently, new tools need to be developed in order to offer more sustainable solutions to assist waste management.

\section{Introduction}

La production industrielle ayant connu une effervescence sans précédent depuis plus d'un siècle, la consommation d'énergie et de matières premières a de ce fait subi une augmentation significative (Ljunggren, 2003 ; Bromley, 200 I). Sachant que les ressources naturelles ne sont pas inépuisables et que les activités d'extraction, de fabrication et de gestion des déchets sont responsables des principaux problèmes de pollution, des études ont démontré que la solution repose en grande partie sur une saine gestion des matières résiduelles. En ce sens, le concept de l'écologie industrielle, présenté pour la première fois en 1989 par Robert Frosch (Frosch et al., 1989), propose une approche systémique qui vise à contrer les phénomènes d'épuisement des ressources naturelles et d'accroissement des volumes de déchets ou résidus produits.

Plus particulièrement, l'écologie industrielle est une approche de gestion intégrée qui tient compte de l'utilisation de l'énergie, des matières premières et des capitaux dans toutes les activités de l'entreprise, que ce soit dans la conception, la production, ou même dans la commercialisation. Suivant la même idée que celle proposée par les 3RV (réduction, réutilisation, recyclage, valorisation), l'écologie industrielle vise l'optimisation des ressources, la réutilisation et la valorisation des résidus, tout en développant une gestion de l'information et l'amélioration des mécanismes de production. L'équilibre environnemental et la croissance économique désirés doivent se faire avec la participation active et la mise en réseau des différents intervenants, puis en considérant les entreprises qui pourraient potentiellement contribuer au système recherché.

Le concept de la gestion intégrée des matières résiduelles vise également l'atteinte de bénéfices environnementaux, 
d'optimisations économiques ainsi que d'acceptabilité sociale. Pour assister les décideurs et les législateurs dans leurs processus de prise de décisions relativement à la gestion des matières résiduelles, de nombreux outils sont désormais proposés. Malgré la popularité croissante de ces différents outils, certains obstacles nuisent à la mise en application de ceux-ci. Dans le but de faire la lumière sur la situation actuelle quant à la gestion intégrée des matières résiduelles, le présent article porte sur les outils d'aide à la prise de décisions visant à favoriser l'écologie industrielle.

\section{Le contexte industriel}

La montée de l'intérêt envers le respect de l'environnement et envers la gestion des matières résiduelles a mené à l'élaboration de différents concepts. II n'existe aucune théorie ultime, mais la connaissance, l'agencement et la mise en application de certains de ces concepts sont d'excellentes approches pour atteindre, de façon responsable, les objectifs visés. En ce sens, le marché de la récupération des matières résiduelles connaît une croissance importante depuis les deux dernières décennies. Pour la gestion des résidus, plusieurs voies sont possibles. En fonction des technologies, des contraintes et des disponibilités, il peut parfois s'avérer complexe de déterminer quel mode de gestion s'avère être le plus durable. Traditionnellement, l'approche favorisée par les individus et les entreprises pour la gestion des résidus solides était de type « end-of-pipe » : les matériaux sont extraits, transformés, utilisés et, pour terminer, ils sont enfouis ou incinérés. Désormais, les efforts portent plutôt sur la préservation des ressources en tentant de réduire la nécessité d'exploiter celles-ci. Pour y parvenir, certaines alternatives permettent de prolonger le cycle de vie des matières, réduisant ainsi la demande en matériau brut. Lorsque les conditions le permettent, le moyen le plus simple de réduire la consommation est tout simplement de réutiliser les matériaux. En addition à la réutilisation, la valorisation de ces matières dans les cycles industriels de fabrication a aussi pour effets de diminuer l'exploitation des ressources naturelles ainsi que la pollution qui en découle (Gouvernement du Québec, 2006 ; Ministère de l'Ecologie et du Développement durable, 2004 ; Finnveden et al., 2005).

Devant le phénomène de mondialisation, les industries doivent donc apprendre à combiner les notions de gestion d'entreprise et de protection de l'environnement dans leurs processus opérationnels. Le contexte économique étant de plus en plus compétitif, les entreprises optent habituellement pour un mode de disposition qui favorise leur rentabilité économique plutôt que d'évaluer les impacts environnementaux et sociaux associés à leurs prises de décisions. L'urgence d'opter pour une telle approche de gestion est alimentée par le fait que le taux de régénération des ressources naturelles est actuellement inférieur aux besoins exprimés (Sundkvist et al., 1999). Cette problématique pourrait toutefois être atténuée par la mise en place d'un réseau collectif de gestion des matières résiduelles. Pour y parvenir, les entreprises doivent connaître quels sont les enjeux et quelle est leur importance dans une optique de gestion saine et écologique. Au Québec, cette problématique est d'ailleurs accentuée en raison du fait qu'il n'existe actuellement aucun mécanisme permettant de centraliser l'information concernant les divers matériaux générés et consommés dans les systèmes industriels. Un tel mécanisme permettrait donc aux entreprises d'évaluer rapidement les possibilités qui s'offrent à elles quant à la gestion de leurs matières résiduelles et faciliterait la synergie nécessaire à l'atteinte d'une écologie industrielle.

Actuellement, la majorité des entreprises gèrent leurs matières résiduelles aléatoirement, au fur et à mesure qu'elles sont générées, et aucun plan d'urgence n'est établi en cas de brisure de stock ou de disposition substantielle de déchets. Ainsi, une crise interne pourrait subvenir suite à un événement inattendu et les répercussions pourraient affecter l'équilibre du système auquel ces entreprises appartiennent, voire l'équilibre des systèmes connexes. Par exemple, les volumes de résidus générés et la demande inattendue de poteaux de bois traité, durant la crise du verglas de 1998, avaient complètement paralysé la province du Québec durant plus d'une dizaine de jours. Cette crise aurait pu être atténuée si un plan de gestion avait été élaboré en fonction des événements chaotiques pouvant survenir.

Vu l'importance des volumes en jeu, la mauvaise gestion des déchets exerce un impact sur la contamination de l'air, de l'eau et des sols, et participe à la destruction de plusieurs espèces végétales et animales. L'accroissement constant des volumes enfouis contribue à alourdir le poids de l'empreinte écologique occasionné par la multiplication des lieux d'enfouissement sanitaire et des dépôts de matériaux secs. Dans le but de favoriser la conservation des ressources et de protéger les milieux naturels, la Commission sur la gestion des matières résiduelles permet de considérer certains déchets comme une source de matière première (BAPE, 1997). Bien que l'approche systémique de l'écologie industrielle tente de réduire le poids de l'empreinte écologique causé par les entreprises, ces dernières gèrent majoritairement leurs déchets de façon indépendante (Minck, 1995). Cette problématique a rapidement mené à une augmentation importante du volume de matériaux recyclés, alors que d'autres modes de valorisation sont disponibles. Conséquemment, plusieurs décideurs et citoyens commencent à se questionner sur le point de savoir si le recyclage est réellement la meilleure solution (Bjorklund et al., 2005).

Quoique les progrès technologiques aient facilité la prolifération de divers modes de valorisation des résidus, les filières disponibles demeurent généralement limitées. Devant l'accroissement du volume de matières résiduelles générées, il devient donc essentiel d'évaluer la possibilité d'investir dans la mise en place de nouveaux procédés de valorisation. De plus, vu les impacts associés à la récupération et au recyclage des différents matériaux, il pourrait s'avérer plus avantageux, dans une optique de développement 
durable, d'opter pour d'autres voies. Malgré les récents efforts pour hiérarchiser les modes de disposition, il est à noter que les conditions géographiques, technologiques, environnementales, économiques et sociales influencent significativement la pertinence de prioriser un mode plutôt qu'un autre, et qu'il est actuellement impossible de comparer des scénarios de façon efficiente.

\section{Les outils de gestion}

Dans le cadre d'un programme de gestion responsable des matières résiduelles, l'évaluation des avantages et inconvénients offerts par les différentes alternatives d'élimination demande une attention et une objectivité particulières. Pour y parvenir, de nombreux outils sont disponibles afin d'appuyer les décideurs dans leurs prises de décisions. Actuellement, l'outil favorisé s'avère être l'analyse de cycle de vie (ACV ; Korhonen et al., 2004). Les outils de calculs de flux, les analyses de la sensibilité et les systèmes d'informations géographiques (SIG) comptent aussi parmi la liste des mécanismes d'aide à la gestion des matières résiduelles. Afin d'obtenir des informations fiables concernant les émissions de polluants et leurs impacts sur l'environnement, l'approche de l'ACV s'avère efficace pour comparer différents scénarios envisageables (Thomas et al., 2005).

Bien que des outils, tel que l'ACV, soient utilisés pour accompagner la prise de décisions, ceux-ci comportent tout de même des lacunes quant à l'interdépendance entre les variables, pouvant ainsi influencer significativement les résultats (Petersen et al., 2005). Notamment, les systèmes étudiés à l'aide de ces outils sont linéaires et rigides, les fluctuations dans la disponibilité des ressources et dans la productivité sont négligées, la cinétique des transports n'est pas considérée, la synergie inter-industries n'est pas favorisée, et enfin le choix du mode de valorisation doit être déterminé dès le départ. En ce sens, il est essentiel de prendre en considération les flux de matières, les opérations de collecte, puis les méthodes de traitement et d'élimination des résidus (Thomas et al., 2005). De plus, les valeurs greffées aux variables sont fixes, alors qu'en réalité ces dernières fluctuent continuellement (Costi et al., 2004). Les outils actuels servent principalement à comparer différents scénarios établis. Ainsi, l'ACV ne permet pas d'effectuer efficacement des simulations aux fins d'optimisation.

Le fonctionnement des outils disponibles cause aussi problèmes. Les utilisateurs critiquent la complexité et la lourdeur de ceux-ci, obligeant ainsi les entreprises à posséder l'expertise nécessaire à leur emploi et à l'interprétation des résultats. De plus, ces outils demandent habituellement l'intégration de nombreuses variables, ce qui ralentit considérablement le processus de prises de décisions. Pour ces raisons, plusieurs décideurs choisissent plutôt de prendre leurs décisions de façon instinctive.

\section{Discussion}

Le but de la gestion responsable des matières résiduelles est de trouver de nouveaux débouchés pour les déchets de la société de façon environnementalement efficiente, économiquement abordable et socialement acceptable. Pour atteindre cette durabilité, les outils doivent permettre de prédire convenablement la charge infligée à l'environnement suite à l'application d'un système de gestion des matières résiduelles (Thomas et al., 2005). Dans un contexte de gestion environnementale, les gestionnaires peuvent choisir de maintenir leur adhésion aux paradigmes qui leur servent depuis déjà plus d'une trentaine d'années, ou plutôt choisir de favoriser les nouvelles approches systémiques qui favorisent l'adaptation aux conditions évolutives (Emison, 1996 ; Deco et al., 1997).

Bien qu'il doive s'y installer une synergie entre les industries présentes dans un système commun, l'indépendance de chacune des entités occasionne un morcellement dans la gestion des matières résiduelles. Or les résidus disposés sont habituellement dirigés vers différents modes d'élimination d'une façon linéaire (" end of pipe »; Wang et al., 1996). En regroupant les volumes disposés vers un système commun de gestion, l'effet de masse permettrait possiblement de rediriger les divers flux de matières vers un réseau partagé, ce qui offrirait un plus grand éventail de filières de valorisation potentielles. Toutefois, un mécanisme doit être mis en place afin de catégoriser et caractériser les modes de valorisation disponibles. Ainsi, il serait possible d'élaborer des scénarios de gestion de façon à régulariser le réseau systémique, établir une liste de priorités en cas de rupture de stock, prévoir des réservoirs en cas d'augmentation subite du volume à redistribuer, etc.

En raison des fluctuations constantes dans les volumes de matières résiduelles générées par les industries, il est impossible d'élaborer un mécanisme pour évaluer avec justesse les variations futures (Hilborn, 2000). Comme les entreprises, dans une optique d'écologie industrielle, sont interdépendantes et s'alimentent entre elles similairement aux éléments d'un écosystème, chacune des fluctuations de variables exerce potentiellement une influence sur l'ensemble du système. De plus, sachant que la présence d'une seule variable aléatoire, dans un système complexe non linéaire, entraîne l'ensemble de ce système vers un déséquilibre, il devient essentiel de vérifier quelles variables peuvent engendrer ce déséquilibre et de déterminer si ces variables sont déterministes ou stochastiques.

Un des principaux obstacles à la gestion des matières résiduelles, pour les décideurs, les professionnels et le législateur, réside dans le caractère évolutif des systèmes qui fait en sorte que les décisions actuelles ne représentent pas nécessairement les meilleures solutions face aux conditions futures. II est donc impensable, dans une optique de développement durable et face aux fluctuations constantes, 
d'opter pour une filière précise d'élimination ou de valorisation des résidus et d'espérer l'appliquer indéfiniment. Ainsi, les prises de décisions doivent s'adapter aux conditions réelles de façon à réduire les impacts sur l'environnement, à favoriser la croissance économique et à satisfaire les exigences sociales (Emison, 1996).

La prise de décisions demande un certain niveau de connaissances et d'expertise pour examiner adéquatement les informations qui sont souvent imprécises, incomplètes, biaisées, voire erronées (Jagielska, 1998). Le caractère aléatoire régissant la gestion des matières résiduelles, les erreurs découlant de l'approximation des valeurs paramétriques et les ambiguiités dans la planification et dans les contraintes représentent des sources d'incertitudes qui exercent une influence significative sur l'équilibre d'un système (Chang, 1998).

Suite à ces observations, il apparaît juste d'affirmer qu'une nouvelle approche pour la gestion des matières résiduelles doit être élaborée. Cette dernière devrait viser le resserrement de la cohésion interindustrielle recherchée dans l'optique d'une écologie industrielle. Pour y parvenir, de nouveaux outils adaptifs et évolutifs devront être élaborés de façon à respecter davantage l'objectif du développement durable.

\section{Conclusion}

La réussite de l'application d'un système de gestion de matières résiduelles, dans un système industriel, est directement liée à l'application d'outils de contrôle simples et efficients. Le but de la gestion responsable des matières résiduelles est de trouver de nouveaux débouchés pour les déchets de la société de façon environnementalement efficiente, économiquement abordable et socialement acceptable. Pour atteindre cette durabilité, les outils offerts doivent permettre de prédire convenablement la charge infligée à l'environnement suite à l'application d'un système de gestion des matières résiduelles (Thomas, 2005). En ce sens, les modèles et les outils de gestion qui seront développés dans l'avenir devront reposer sur des concepts universels de la gestion. Ainsi, il serait éventuellement possible d'adapter ces modèles et ces outils afin de les appliquer à la gestion de n'importe quel résidu, indépendamment du lieu géographique et des conditions sociales, économiques ou environnementales.

Actuellement, la nouvelle tendance qui s'installe quant à la gestion des résidus vise à favoriser l'élaboration de systèmes globaux de gestion des matières résiduelles plutôt que de traiter la gestion des résidus selon un type spécifique de matière. En outre, il a été démontré à maintes reprises que le choix le plus économique n'est pas nécessairement le choix le plus environnemental, et que la meilleure option en termes d'équité sociale n'est pas nécessairement la plus économique ou la plus environnementale (Wang, 1996). Ainsi, en intégrant les notions d'écologie industrielle à une approche systémique non linéaire de type réseau, il deviendrait possible de répondre à ces critères fondamentaux.

Une toute nouvelle approche de gestion des résidus fondée sur la dynamique des systèmes, développée dans une vision de développement durable, permettrait alors des retombées positives tant pour les utilisateurs, pour les législateurs et pour l'ensemble de la société. De plus, une des principales retombées découlant d'une telle approche serait une diminution du volume de déchets enfouis ou incinérés, puis une réduction de la nécessité d'extraire des ressources non renouvelables (Craighill, 1996).

\section{Références}

Albino, V. (2002). Input-output models for the analysis of a local/global supply chain. International Journal of Production Economics. 78, p. $119-131$.

Bureau des audiences publiques en environnement (1997). Déchets d'hier, ressources de demain. Gouvernement du Québec. 504 p.

Bjorklund, A. and G. Finnveden (2005). Recycling revisited - Life cycle comparisons of global warming impact and total energy use of waste management strategies. Resources, Conservation and Recycling. 44, p. 309-317.

Bromley, P. (200 I). Taking energy management into the boardroom. Manufacturing Engineer. 80, p. 55-58.

Brümmerhoff, D. (1996). Finanzwissenschaft. München, Allemagne, Vahlen, $7^{\mathrm{e}}$ édition

Chang, N.-B. (2000). Siting recycling drop-off stations in urban area by genetic algorithm-based fuzzy multiobjective nonlinear integer programming modeling. Fuzzy Sets and Systems. I | 4, p. I33- 149.

Costi, P., R. Minciardi, et al. (2004). An environmentally sustainable decision model for urban solid waste management. Waste Management. 24, p. 277-295.

Craighill, A. L. and J. C. Powell (1996). Lifecycle assessment and economic evaluation of recycling: a case study. Resources, Conservation and Recycling. 17, p. 75-96.

Deco, G., C. Schittenkopf, et al. (1997). Information flow in chaotic symbolic dynamics for finite and infinitesimal resolution. International Journal of Bifurcation and Chaos in Applied Sciences and Engineering. 7, p. 97-105.

Emison, G.A. (1996). Chaos theory, fuzzy logic and systems thinking: pathways to synergistic environmental management. Proceedings of the 1996 Air \& Waste Management Association's 89th Annual Meeting \& Exhibition, Nashville, TN, USA, Air \& Waste Management Assoc, Pittsburgh, PA, USA.

Finnveden, G., J. Johansson, et al. (2005). Life cycle assessment of energy from solid waste - Part I: General methodology and results. Environmental Assessments and Waste Management 
Journal of Cleaner Production. 13, p. 213-229.

Frosch, Robert A., and Nicholas E. Gallopoulos (1989). Strategies for Manufacturing. Scientific American. 26I, p. I44- 152.

Gouvernement du Québec (2006), Loi sur la qualité de l'environnement, L.R.Q., chapitre Q-2.

Harrison, K.W., R. D. Dumas, et al. (200 I). Decision support tool for life-cycle-based solid waste management. Journal of Computing in Civil Engineering. I5, p. 44-58.

Hilborn, R. (2000). Chaos and Nonlinear Dynamics, Second ed. London, U.K.: Oxford Univ. Press. 650 p.

Jagielska, I. (1998). Hybrid rough sets - Neural network approach to the development of a decision support system. IEEE World Congress on Computational Intelligence. p. 24-28

Kao, J.-J. (1996). IAC network for composition of waste-incineration facility. Journal of Computing in Civil Engineering. 10, p. 168-171

Knaus, M., Löhr, D., O'regan, B. (2006). Valuation of ecological impacts: A regional approach using the ecological footprint concept. Environmental impact assessment review. 26, p. I 56-I69

Korhonen, J., L. Okkonen, et al. (2004). Industrial ecosystem indicators - direct and indirect effects of integrated waste- and by-product management and energy production. Clean Technologies and Environmental Policy. 6, p. I62-173.

Ljunggren Soderman, M. (2003). Including indirect environmental impacts in waste management planning. Resources, Conservation and Recycling. 38, p. 213-24I.

Meadows, D., Randers, J. et Behrens, W. (1972). Limits to growth, Universe Books, New-York. 309 p.

Minck, G. H. (1995). Creating platforms for environmental technology development. Proceedings of the 1995 IEEE International Symposium on Electronics and the Environment, Orlando, FL, USA.

Ministère de l'écologie et du développement durable (2004). Prévention de la production de déchets. République Française, Paris. 3 I p.

Neves, J., Cortez, P. (1997). An artificial neural network: Genetic based approach for time series forecasting. $4^{\text {th }}$ Brazilian Symposium on Neural Networks, p. 9-13

Nunez, M., R. Morales, et al. (2002). Automatic discovery of rules for predicting network management events. IEEE Journal on Selected Areas in Communications. 20, p. 736-745.

Petersen, A. K. and B. Solberg (2005). Environmental and economic impacts of substitution between wood products and alternative materials: A review of micro-level analyses from Norway and Sweden. Forest Policy and Economics. 7, p. 249-259.

Rees, W.E. (1996-A). Revisiting carrying capacity: Area-based indicators of sustainability. Population and environment. 17, p. 195-215.

Rees, W.E., Wackernagel, M. (1996-B). Urban ecological footprints: Why cities cannot be sustainable, and why they are the key to sustainability. Environmental impact assessement review. 16 , p. 223-248.

Riggle, C. and G. Madey (1997). An analysis of the impact of chaotic dynamics on management information flow models. European Journal of Operational Research. 103, p. 242-54.

Sundkvist, A., A. Jansson, et al. (1999). Energy flow analysis as a tool for developing a sustainable society - a case study of a Swedish island. Resources, Conservation and Recycling. 25, p. 289-299.

Thomas, B. and F. McDougall (2005). International expert group on life cycle assessment for integrated waste management. Environmental Assessments and Waste Management Journal of Cleaner Production. 13, p. 321-326.

United Nations development program (annual). Human development report, Oxfortd University Press, New-York.

Wackernagel, M., Yount, J.D. (1998). The ecological footprint: An indicator of progress toward regional sustainability. Environmental monitoring and assessment. 51, p. 51 I-529.

Wang, F. S., A. J. Richardson, et al. (1996). SWIM - a computer model for solid waste integrated management. Computers, Environment and Urban Systems. 20, p. 233-246.

W.C.E.D. (1997). Our Common Future. World commission on environment and development, Gro Harlem Bruntland chair, Oxford University Press, New-York.

Wilson, J. A. and R. Alden (1994). Chaos theory, complexity: Fishery management solutions?. Sea Technology. 35, p. 61-63.

World Resources Institute (1994). World Resources 1994-95, Oxford University Press, New-York.

World Resources Institute (2005). World Resources 2004-05, Oxford University Press, New-York.

Zhao, S., Z. Li, et al. (2005). A modified method of ecological footprint calculation and its application. Ecological Modelling. I85, p. 65-75. 